\title{
ALMOST UNIFORM CONVERGENCE VERSUS POINTWISE CONVERGENCE
}

\author{
JOHN W. BRACE ${ }^{1}$
}

In many an example of a function space whose topology is the topology of almost uniform convergence it is observed that the same topology is obtained in a natural way by considering pointwise convergence of extensions of the functions on a larger domain $[1 ; 2]$. This paper displays necessary conditions and sufficient conditions for the above situation to occur.

Consider a linear space $G(S, F)$ of functions with domain $S$ and range in a real or complex locally convex linear topological space $F$. Assume that there are sufficient functions in $G(S, F)$ to distinguish between points of $S$. Let $S_{\beta}$ denote the closure of the image of $S$ in the cartesian product space $\times\{\overline{g(S)}: g \in G(S, F)\}$. Theorems 4.1 and 4.2 of reference [2] give the following theorem.

THEOREM. If $g(S)$ is relatively compact for every $g$ in $G(S, F)$, then pointwise convergence of the extended functions on $S_{\beta}$ is equivalent to almost uniform converqence on $S$.

When almost uniform convergence is known to be equivalent to pointwise convergence on a larger domain the situation can usually be converted to one of equivalence of the two modes of convergence on the same domain by means of Theorem 4.1 of [2]. In the new formulation the following theorem is applicable.

In preparation for the theorem, let $B(S, R)$ denote all bounded real valued functions on $S$ which are uniformly continuous for the uniformity which $G(S, F)$ generates on $S . G(S, F)$ will be called a full linear space if for every $f$ in $B(S, R)$ and every $g$ in $G(S, F)$ the function $f g$ obtained from their pointwise product is a member of $G(S, F)$. ( $S, G)$ denotes $S$ with the weakest topology such that each $g$ in $G(S, F)$ is continuous, while $(S, G) \cup\{\infty\}$ denotes the one point compactification of $(S, G)$.

Theorem. If $G(S, F)$ is a full linear space in which pointwise convergence and almost uniform convergence are equivalent then $(S, G)$ is

Presented to the Society, January 22, 1959; received by the editors November 16, 1959 and, in revised form, February 15, 1960.

1 The author is indebted to the referee for his helpful suggestions.

This research was supported in part by the Air Force Office of Scientific Research AF 18(603)-78 and in part by the National Science Foundation, Grant 9414. 
either (i) compact, or (ii) locally compact and each $g$ in $G(S, F)$ can be continuously extended over $(S, G) \cup\{\infty\}$ such that $g(\infty)=0$.

Proof. Let $C S$ be the compactification of $(S, G)$ obtained by taking the closure of the image of $S$ in $\times\{\overline{f(S)}: f \in B(S, R)\}$. Assume $(S, G)$ is not compact and that there is an $s_{0}$ in $C S-S$, a net $\left\{s_{\alpha}\right\}$ in $S$, and a function $g$ in $G(S, F)$ such that the net $\left\{s_{\alpha}\right\}$ converges to $s_{0}$ but the net $\left\{g\left(s_{\alpha}\right)\right\}$ does not converge to 0 . There exists a net $\left\{f_{\delta}\right\}$ in $B(S, R)$ converging pointwise on $S$ to the function which is identically one while all continuous extensions of the members of the net have the value zero at $s_{0}$. This gives a contradiction in that the net $\left\{f_{\delta} g\right\}$ in $G(S, F)$ converges pointwise to $g$ but not almost uniformly. Thus when $(S, G)$ is not compact each $g$ in $G(S, F)$ can be continuously extended over $C S$ so that $g(s)=0$ for every $s$ in $C S-S$. Since $C S$ is the Hausdorff completion of $(S, G)$, there is only one point in $C S-S$. Thus $(S, G)$ is locally compact and the proof completed.

Corollary. A uniform space $E$ is compact if and only if pointwise convergence and almost uniform convergence are equivalent in $C(E, R)$, all uniformly continuous real valued functions on $E$.

Proof. If $E$ is compact, see Theorem 4.2 of [2]. For the converse, observe that each $f$ in $C(E, R)$ is bounded, Theorem 1.3 of [2]. Thus $f g$ is in $C(E, R)$ whenever $f$ and $g$ are, and $C(E, R)$ is a full linear space. Since $C(E, R)$ contains nonzero constant functions, conclusion (ii) of the second theorem is not possible and $E$ must be compact.

\section{BIBLIOGRAPHY}

1. J. W. Brace, Almost uniform convergence, Portugal. Math. vol. 14 (1955) pp. 99-104.

2. - The topology of almost uniform convergence, Pacific J. Math. vol. 9 (1959) pp. 643-652.

UNIVERSITY OF MARYLAND 